\title{
The Problem of Interaction Between the Old and the New When Digitalizing Education
}

\author{
Shpeka K.A. \\ Ural State Pedagogical University, Yekaterinburg, Russian Federation \\ Email: kshpeka@mail.ru
}

\begin{abstract}
The article deals with the problem of the transition of Russian education into digital form, identifies the objective features of this process, which create the need to create a digital telecommunication educational environment, identifies the main points of the education process, which enable to consider digitalization in various aspects in the implementation of the education process. An attempt is made to reveal the contradiction between the new and the old in the sense of using traditional methods and means of training and new information technologies based on the digitization of the communication process. It is established that a number of humanities disciplines require the use of traditional methods and forms of education, while increasing the share of digital tools used as methodological support. The problem of forming the correct content of the information content of training courses in the form of user-oriented educational information products is identified. The problem of interaction of digital learning tools (electronic textbook) and traditional ones (paper textbook) is touched upon. It is concluded that it is necessary to reproduce traditional methods and means of education in the digitization of education in the study of a number of humanities.
\end{abstract}

Keywords: digitalization of education, electronic learning resources, telecommunication technologies in

education, Internet, information product, electronic textbook, electronic means of communication

\section{INTRODUCTION}

The reality of our time is that digital technologies have become an integral part of modern life. Some people have expressed concerns that various digital technologies are becoming not just a part of a person's life in the $21 \mathrm{st}$ century, but even a part of themselves. It is arguable, but the fact is that without digital technologies and telecommunications based on them, modern life is simply unthinkable. And this is true both in relation to the information and cultural environment of human existence, and the production environment, where man is involved as a producer of various goods (both material and spiritual) intended for public consumption.

\section{METHODS OF RESEARCH}

This article is an attempt to understand the principles of the transition of Russian education to digital form on the basis of the philosophical and dialectical method, as well as general scientific methods - analysis and synthesis.

\section{RESULTS AND DISCUSSION}

Of course, education, including higher-level education, cannot be left out of the process of digitalization. What is meant by digitalization of education? To understand it, we should pay attention to a number of fundamental points that reveal the essence of education as a process.

In my opinion, such moments are:

first, the process of teacher-student interaction; second, the environment that mediates this interaction; third, independent work of the student on mastering knowledge, during which the level of performance and practical skills necessary for the future profession is formed. Effective education is impossible without independent work.

The first point of principle needs no special explanation. Interaction here is, first of all, communication about the assimilation of the material intended for study. The teacher acts as a mentor, the student as a mentee. The interaction is about learning, i.e. setting cognitive problems and getting new knowledge about the world and about themselves in this world.

As for the environment in which this communication takes place, it is a kind of subsystem that includes the social environment (micro-collective), as well as information products that communicate between students and teachers. The nature, scope and availability of information depend 
on the goals set for the learning process. It seems to me that information, as a component of the educational environment, should be systematized, ordered and available in accordance with these goals. The goals, in turn, are determined by the specifics of social relations of a particular historical epoch. As a rule, in the educational environment, the specifics of such relations take the form of specific ideological values, according to which information exchange between participants in the educational process is built. Whether we like it or not, in any case education serves the interests of society, so the ideological element in its content will always be present. The third component is designed to determine the degree of formation of the student to independently solve cognitive and practical issues that arise during training or may arise, as well as those that may appear in any professional activity in future. The level of such formation depends on the volume and availability of information products necessary to solve the problem situation (in the case of training, this will be a cognitive problem as a discrepancy between knowledge about the phenomenon being studied and ignorance about it), as well as on the ability to analyze these information products. In this regard, I agree with the opinion of $M$. S. Kudlaev: "During the rapid development of science, many corporations require employees who are ready to work with the latest technologies at all levels of their production, and there is no need for employees who do not possess special skills" [Kudlaev, 2017].

In the light of the three main points identified above, we can say that digitalization of education is the introduction of electronic communication devices into the learning process that enable to receive and process educational information at the right speed, as well as contribute to improving the effectiveness of interaction between teachers and students.

Of course, from this point of view, digitalization requires the widespread introduction of computers, appropriate software and an electronic communication network into the educational environment, allowing participants to interact with each other in the educational process on the one hand, and to facilitate the circulation of information products between participants on the other. Today, it can be stated that most universities have already introduced electronic means of communication in the educational process, which is carried out within the framework of almost all educational programs. This form of interaction between a student and a teacher, such as online lectures, seminars, and courses, is widely spread. But nevertheless, "Based on the graphs, it is clear that the number of personal computers in educational organizations is growing. But, unfortunately, technologies are being implemented in higher education institutions much more slowly than the digitalization of production. Insufficient level of development of educational information technologies is also observed in general education institutions: often the insufficient level of development of skills of school leavers is due to training on outdated equipment or its complete absence" [Kudlaev, 2017]. In this regard, we should agree with the interpretation of the process of digitalization itself as a kind of social situation in which a person objectively must move to digital forms of interaction, "this is an objective process of displacing all the analogue from technology, economy, culture" [Safuanov R. M., Lekhmus M. Yu, Kolganov E. A., 2019, p.109]. According to various estimates, the number of communication devices currently involved in the Internet reaches 25 billion units, which exceeds even the number of users [ibid.]. The process of dissemination of digital communication technologies is so broad that the objectivity of its nature is not in doubt. Today, digital technologies allow teachers to get the right text easily, edit it for the needs of an educational program or course, and use it in the classroom effectively. Often the material can be visualized and placed on various communication platforms, which can be created even on the basis of social networks (for example, almost every group of students today has its own page in the "VK" social network).

The new social situation associated with new communication technologies has been called "Industry 4.0.", and at the same time, when it comes to the essence of the new technological order, it is emphasized that "this is a fundamentally new approach to determine the properties of all human things, as well as the methods of their production and consumption" [Shpurov I.]. This, in turn, creates a whole range of risks associated with the uncertainty of the consequences of the transition of education to digital "rails". Although this transition also promises significant advantages. These include, first of all, an increase in the proportion of students' independence in the learning process, which can increase their readiness to practice in a particular professional field. This is a definite plus, and perhaps the biggest one. Also, the digitalization guarantees economizing traditional information carriers, i.e., decreases the need for acquiring and publishing paper textbooks, workbooks, notebooks, etc. Among the possible drawbacks there are: lack of creativity in teaching (it is more difficult for a person to show their individuality when working with digital materials), reduced mental activity (easy availability of necessary materials "kills" the need to construct a response from numerous "grains"), poor socialization (ease of interaction between the teacher and the student avoiding the micro-collective), poor physical development, high control over individuals as users of electronic communication devices, the reduced role of the teacher [Digitalization of education]. In this regard, one of the most urgent tasks of the transition to a new digital form of Russian education is the creation of new education management systems that will make it more flexible, as well as improving the professionalism of teachers themselves in the field of digital technologies [Meeting the challenge of change: seven tasks of digitalization of Russian education]. But most importantly - it is simply not possible to predict all the consequences of this transition. Therefore, a sharp rejection of traditional forms of education in this regard is not considered acceptable by the author of the article. In addition, in this regard, electronic educational resources 
are now widely used among teaching tools. These include scientific and pedagogical, educational and methodological, regulatory, instructional and technological information that is presented in electronic forms, and, accordingly, in special software for educational purposes. This is due to the following circumstances.

First, it is the need to provide citizens with affordable high-quality education that meets the requirements of modern society and the state. Therefore, each teacher faces the need to create and subsequently update an electronic complex of educational and methodological support for the taught discipline, which should contribute to improving the effectiveness of education. The fact is that modern students rarely visit libraries, they prefer to work with digital devices, getting all the necessary information from there. Therefore, it is necessary to solve modern didactic tasks based on the actual situation, and not the required one.

Second, constant changes in the sphere of public relations also lead to the need to master new hardware and software quickly, and, most importantly, new information products. Mastering technologies for obtaining and processing information through the telecommunication environment is impossible without appropriate training tools.

The above mentioned things point to the great importance of creating a digital information environment associated with the organization of the educational process in various academic disciplines with the use of electronic educational resources. However, the problem is complicated by the fact that a number of humanities subjects are difficult to digitalize in the sense that teaching them requires familiarity directly with the texts of primary sources. Working with them requires a long time, hard work and a strong tension of thinking. These are philosophy, literature, culture studies, etc. It is not an exaggeration to say that the study of, for example, philosophy by schematizing the content of sources, is hardly possible. The main element in the assimilation of philosophical categories is individual thinking. However, the methodological component, which includes recommendations and adjustments to the process of individual work with sources, can only benefit if it takes the form of digital interaction between the student and the teacher. In addition, modern Internet tools make it possible to automate this process, preserving the possibility of direct communication between the subjects of the educational process. For example, electronic versions of educational and methodological complexes posted on the Internet on specially created communication platforms do not exclude the possibility of direct communication at lectures, as well as prompt consultation of students, if necessary, which even contributes to improving the effectiveness of studying such humanities. Therefore, recognizing the importance of digitalization of modern communication processes and the widespread introduction of digitalization in education, we should say, that even in the digital information and communication environment, an important element remains, that is abstract humanities, and it requires the predominant reproduction of traditional approaches to learning, but with the use of new information technologies as organizational and methodological tools. These tools should make it possible to modernize the form of interaction between teachers and students, as well as fill the educational environment with the necessary amount of information products (the author of these lines still remembers the times when a student engaged in research work had to request the necessary rare literature on interlibrary subscription in the form of microfilms, which significantly complicated the learning process). However, it is important to consider the following. When the information flow associated with its availability increases, the amount of information delivered (having the ability to being delivered) to the consumer in the form of certain products, which ultimately have the form of text (although in some cases the text can be adapted to the perception of a specific audience using visualization methods) increases. However, the density and availability of information flows has a negative impact on the quality of the information received, since it becomes more difficult to analyze information due to the oversaturation of the flow with texts of low information content. The situation is the reverse of the shortage of information products valuable information becomes lost in the vast information flow. Therefore, one of the urgent tasks of digitalization of the educational environment is to filter information products based on the criterion of their informativeness within specific educational programs or individual courses. Without this education cannot be effective, and we will only get a situation of information hunger in its opposite form (the lack of information available for analysis is bad, its overabundance is also bad, because the student is forced to spend maximum time to select the information necessary in terms of the cognitive task). Therefore, the literature notes the need to create special methods for selecting web pages to include them in specialized web archives dedicated to specific cognitive issues. Such web archives will allow users to orient themselves as accurately as possible in the search and processing of information, i.e. they will be created due to their interests, focusing on the specifics of a person as a subject of knowledge [Colin P., 2017].

In this regard, the concept of "electronic educational resources" has entered scientific and pedagogical circle. It is defined and interpreted by many researchers and practitioners in different ways. Taking into account a wide variety of definitions and interpretations it is possible to isolate the following as a quintessence: these are educational materials that are reproduced and transmitted using electronic devices.

According to the interstate standard R 53620-2009, an electronic educational resource is a resource presented in electronic and digital form and that includes the structure, subject content and metadata about them. An electronic educational resource may include data, information, and software necessary for its use in the learning process.

It can also be understood as a set of scientific and pedagogical, educational and methodological, regulatory, instructional and technological information presented in 
various electronic formats and various software tools and educational systems used for their use.

Electronic educational resources are also referred to as educational materials that are reproduced using electronic devices. All this allows us to define them as a set of software, information, technical and organizational support, electronic publications, placed on machinereadable storage devices and / or on the web. It turns out that these are specially formed blocks of various information resources (sources and tools) intended for use in the educational process, for the reproduction and functioning of which computer equipment is necessary. Summarizing, we can conclude that an electronic educational resource is a learning tool presented in electronic form or on electronic storage device, which can combine different types of information and be created using various software, as well as transmitted between participants of the educational process through electronic communication networks.

There is a need to create such an educational information product that would contain all the advantages of the information resource as a whole and would serve as a kind of tool for mastering the content of the course being studied. In this regard, we come to the problem of an electronic textbook. Whatever we consider the problem of digitalization, it should be recognized that the main means of successful learning with an increasing share of independence of the student is educational literature, or simply put - a textbook.

The problem of creating electronic textbooks, which together with online courses, consultations and an electronic system of assessment and control of the received knowledge, and allowing to transit education into a digital format, is very important at the present time. In order to do this, it is necessary to determine what such a

\section{CONCLUSION}

Considering the above mentioned things it is possible to draw the following conclusions. Currently, digitalization of education is necessary. It should involve the creation of a system for transmitting educational data in digital form between the student and the teacher, as well as the exchange of data between other participants in the educational environment. The educational environment should gradually become digital. At the same time, a number of humanities subjects largely require the preservation of traditional methods and forms of

\section{REFERENCES}

[1] Kudlaev, M. S. (2017), "The process of digitalization of education in Russia" ["Protsess tsifrovizatsii obrazovaniya V Rossii"], Molodoy ucheniy, No. 31, pp. 3-7.

[2] Marchenko, M. (2017) "The main advantage of a paper book over an electronic one" ["Glavnoye textbook should be, i.e. what characteristics it should have to replace a traditional book. Currently, the advantage of electronic texts over traditional paper carriers is being actively discussed. At the same time, it is noted that electronic information resources mainly focus on the idea of generalized information content, rejecting particular manifestations of the material carrier in the form of certain of its features, which allow the student to project the knowledge obtained from the book on certain features of material reality [Patricia Kay Galloway, 2017]. After all, despite the new social situation based on digital technology (automation of production and exchange), a person continues to be a material being, so it is easier, and perhaps clearer to work with a material information carrier [Marchenko M.]. In addition, texts fixed on paper still have a number of significant advantages that digital carriers are not yet able to reproduce [Advantages of a printed book]. The ability to reproduce these advantages of a paper book is a very debatable issue. One of the advantages of an ordinary book that cannot be digitalized is the ability to visualize, i.e. visualize semantic blocks, since separate pages are perceived by the mind as bricks of one immaterial structure. In addition, the ability to leaf back several times and compare separate sentences contributes to the assimilation of logical connections between the semantic blocks of the text being studied, and this, in turn, makes the acquisition of complex cognitive contexts more qualitative. Electronic means are not yet able to completely replace a paper book in this aspect. So, despite digitalization, the presence of traditional educational literature in the process of education is necessary, but the share of such literature, of course, may not be the same as before.

education. In their case, digitalization should influence the organizational and methodological side of the learning process. In the course of digitalization, the role of such an electronic resource as an electronic textbook is important, but it must be recognized that it will not be able to reproduce all the advantages of a paper book, so preserving the old methods and means of teaching in the form of working with a paper book is one of the important requirements for the transition of Russian education to digital form. 
[7] Shpurov, I. (2016), "Industry 4.0" ["Industriya 4.0"], Ekspert online, available at: http:expert.ru/expert/2016/40/industriya-4_0/ (in Russian).

[8] Galloway, P. (2017) "Archiving Digital Objects as Maintenance: Reading a Rosetta Machine", Preservation, Digital Technology \& Culture (PDT\&C), Vol. 46, Issue 1, DOI: https://doi.org/10.1515/pdtc2016-0024.

[9] Post, C. (2017), "Building a Living, Breathing Archive: A Review of Appraisal Theories and Approaches for Web Archives", Preservation, Digital Technology \& Culture (PDT\&C), Vol. 46, Issue 2, DOI: https://doi.org/10.1515/pdtc-2016-0031.

[10] "Readers absorb less on Kindles than on paper, study finds" (2015), The Guardian, available at: https:// www.theguardian.com/books/2014/aug/19/readersabsorb-less-kindles-paper-study-plot-ereaderdigitisation( in English). 